\title{
Pachymic acid protects H9c2 cardiomyocytes from lipopolysaccharide-induced inflammation and apoptosis by inhibiting the extracellular signal-regulated kinase $1 / 2$ and $p 38$ pathways
}

\author{
FANG-FANG LI ${ }^{1,2^{*}}$, YUAN YUAN ${ }^{1,2^{*}}$, YUAN LIU ${ }^{1,2}$, QING-QING WU ${ }^{1,2}$, RONG JIAO ${ }^{1,2}$, \\ ZHENG YANG ${ }^{1,2}$, MENG-QIAO ZHOU ${ }^{1,2}$ and QI-ZHU TANG ${ }^{1,2}$ \\ ${ }^{1}$ Department of Cardiology, Renmin Hospital of Wuhan University; \\ ${ }^{2}$ Cardiovascular Research Institute of Wuhan University, Wuhan, Hebei 430060, P.R. China
}

Received May 25, 2014; Accepted February 6, 2015

DOI: $10.3892 / \mathrm{mmr} .2015 .3712$

\begin{abstract}
Pachymic acid (PA), a lanostane-type triterpenoid and the major component of Poria cocos alcoholic extracts, has various pharmacological effects, including anti-inflammatory, anti-oxidative and anti-apoptotic. However, few studies have investigated the effects of PA on lipopolysaccharide (LPS)-induced H9c2 cell apoptosis and inflammation, or identified the possible mechanisms underlying these effects. In the present study, H9c2 cardiomyocytes were stimulated by LPS and treated with or without PA. The increased mRNA expression levels of tumor necrosis factor- $\alpha$, interleukin (IL)-1 and IL-6 induced by LPS were attenuated following treatment with PA. PA also attenuated LPS-induced apoptosis, as determined by a terminal deoxynucleotidyl transferase dUTP nick end labeling assay, and regulated the LPS-induced protein expression levels of caspase 3,8 and 9. Furthermore, the phosphorylations of extracellular-regulated kinase (Erk)1/2 and p38 in the LPS-treated H9c2 cells were inhibited by PA. These results suggested that treatment with PA prevented the LPS-induced inflammatory and apoptotic response in cardiomyocytes, which may be mediated by inhibition of the Erk1/2 and $\mathrm{p} 38$ pathways.
\end{abstract}

Correspondence to: Mr. Qi-Zhu Tang, Department of Cardiology, Renmin Hospital of Wuhan University, 238 Jiefang Road, Wuhan, Hebei 430060, P.R. China

E-mail: qztang@whu.edu.cn

${ }^{*}$ Contributed equally

Key words: pachymic acid, extracellular-regulated kinase 1/2,p38, inflammation, apoptosis

\section{Introduction}

Lipopolysaccharide (LPS) from Gram-negative bacteria is a potent stimulus for the inflammatory response by inducing the upregulation and release of cytokines (1). Previous studies have demonstrated that LPS can induce inflammation and apoptosis in cardiomyocytes $(2,3)$. Sepsis, characterized by a systemic inflammatory response to bacterial infection and progressive multi-organ dysfunction, is the leading contributor to morbidity and mortality rates in healthcare administrations worldwide (4). Sepsis causes cardiac contractile dysfunction, which is a major cause of morbidity and mortality in patients with sepsis (5). Pro-inflammatory cytokines, induced by bacterial LPS endotoxin, contributes to myocardial dysfunction during sepsis (6-8). Previous reports have identified that a reduced incidence of sepsis improves outcomes and reduces the mortality rates in patients with heart failure $(9,10)$. It has been demonstrated that a reduced systemic inflammatory response improves cardiac function (11).

Pachymic acid (PA), a lanostane-type triterpenoid and major component of Poria cocos alcoholic extracts, has been reported to possess anti-inflammatory and anticancer properties in various models of cancer and inflammation (12). Triterpenoids, extracted from plants, are usually used as a type of medicine in a number of Asian countries (13) for its sedative, diuretic and tonic effects. Previous studies have revealed that PA induces apoptosis in prostate cancer cells and suppresses breast cancer metastasis (14). Another previous study demonstrated that PA stimulates glucose uptake by improving the expression and translocation of glucose transporter type 4 (15). However, the effect of PA on LPS-induced H9c2 cell apoptosis and inflammation remains to be elucidated. The present study investigated whether PA affected LPS-induced H9c2 cell apoptosis and inflammation and the possible mechanisms underlying these effects.

\section{Materials and methods}

Cell culture. The H9c2 cell line was obtained from the Cell Bank of Academy of Science (Shanghai, China). The cells 
were cultured at a density of $1 \times 10^{6}$ cells/well in standard Dulbecco's modified Eagle's medium (\#C11995; Gibco Life Technologies, Carlsbad, CA, USA), supplemented with $10 \%$ fetal bovine serum (\#10099; Gibco Life Technologies) and 1\% penicillin (100 U/ml; \#15140; Gibco Life Technologies) and streptomycin (100 mg/ml; \#15140; Gibco Life Technologies), and were cultured in a $\mathrm{CO}_{2}$ incubator (Sanyo $18 \mathrm{M}$; Sanyo, Osaka, Japan) with $5 \% \mathrm{CO}_{2}$ at $37^{\circ} \mathrm{C}(16)$. The cells cultured between passages three and five were selected for use in the subsequent experiments. Cells were seeded at a density of $1 \times 10^{6}$ cells per well onto 6 -well culture plates for RT-PCR and $5 \times 10^{5}$ cells per well onto six-well culture plates for TUNEL analysis.

Chemicals. PA was obtained from Shanghai Winherb Medical S\&T Development Co. Ltd. (Shanghai, China) and was up to 98\% in purity. The PA was dissolved in dimethyl sulfoxide (DMSO; Sigma-Aldrich, St. Louis, MO, USA) at $50 \mu \mathrm{M}$ and diluted into DMEM containing $<0.1 \%$ DMSO, which was also present in the corresponding controls (normal saline instead of LPS or PA).

Cell viability assay. The cell viability was evaluated using a Cell Counting kit-8 (CCK-8), according to the manufacturer's instructions (17). Briefly, following the indicated treatment, $10 \mu$ l CCK-8 solution was added to each well of a 96-well plate and, following $4 \mathrm{~h}$ incubation with $5 \% \mathrm{CO}_{2}$ at $37^{\circ} \mathrm{C}$, the absorbance was measured at $450 \mathrm{~nm}$ using a Synergy HT microplate reader (Bio-Tek Instruments, Inc., Winooski, VT, USA). The effect of PA on cell viability was expressed as the percentage cell viability compared with the control group, which was set at $100 \%$.

Terminal deoxynucleotidyl transferase dUTP nick end labeling (TUNEL) assay. TUNEL was performed using the in situ Cell Death Detection kit (Roche Diagnostics, Indianapolis, IN, USA), according to the manufacturer's instructions (18). The cells were grown on cover slips in a 24-well plate and, following treatment, the cells were fixed in $4 \%$ paraformaldehyde (Amresco, LLC, Solon, OH, USA) and permeabilized using $0.1 \%$ Triton X-100 (Amresco, LLC). The cells were incubated in $13 \mu l$ (per cover slip) TUNEL reaction mixture (Roche, Basel, Switzerland) for $1 \mathrm{~h}$ at $37^{\circ} \mathrm{C}$. The nuclei were labeled with $13 \mu \mathrm{l}$ (per cover slip) 4',6-diamidino-2-phenylindole (DAPI; Invitrogen Life Technologies, Carlsbad, CA, USA) for $30 \mathrm{sec}$, and DNA fragmentation was quantified using a DX51 microscope (Olympus Corporation, Tokyo, Japan) at high-power magnification (x200). The percentages of TUNEL-positive cells relative to the DAPI-positive cells were counted by an investigator in a blinded-manner. Image analysis was performed using Image Pro-Plus version 6.0 software (Media Cybernetics, Silver Spring,. MD, USA).

Western blot analysis. Western blotting was performed to determine the activation states of the mitogen-activated protein kinase (MAPK) signaling and apoptotic proteins. Cells were lysed in RIPA buffer (Beyotime Institute of Biotechnology, Shanghai, China). The protein quantities from each of the samples were determined by creating a standard curve using a Bicinchoninic Protein Acid assay kit (\#23227;
Thermo Fisher Scientific, Inc., Waltham, MA, USA), followed by normalization of the protein concentration prior to western blotting. The extracted protein $(50 \mu \mathrm{g})$ from each sample were separated on 8-12\% SDS-PAGE gels (Google Biological Technology, Wuhan, China), and the proteins were transferred onto polyvinylidene difluoride membranes (Millipore, Billerica, MA, USA). The membranes were blocked with 5\% non-fat milk powder (Yili Group, Hohhot, China) and were then incubated with the following primary antibodies overnight at $4^{\circ} \mathrm{C}$ : Monoclonal rabbit GAPDH $(1: 1,000$; \#5174); phospho-Erk1/2 (1:2,000; \#4370); and phospho-p38 (1:1,000; \#4511) (Cell Signaling Technology, Inc., Danvers, MA, USA); polyclonal rabbit p38 (1:200; ab7952; Abcam, Cambridge, UK); and phospho-p90RSK (1:1,000; \#9341; Cell Signaling Technology, Inc.); monoclonal rabbit p90RSK (1:1,000; ab32203; Abcam); and cleaved caspase-3 (1:1,000; \#9664); polyclonal rabbit cleaved caspase-9 (1:1,000; \#9509); cleaved caspase-8 (1:1,000; \#9429) (Cell Signaling Technology, Inc.); and $\mathrm{Bad}$ (1:1,000; ab90435; Abcam); monoclonal rabbit Bcl-2 (1:1,000; \#3498); and polyclonal rabbit Bcl-xL (1:1,000; \#2762) (Cell Signaling Technology, Inc.). The membranes were subsequently incubated with secondary antibody (Goat-anti-Rabbit IRDye $800 \mathrm{CW}$ or Goat-anti-Mouse IRDye $800 \mathrm{CW}$; Licor Biosciences, Lincoln, NE, USA) at $25^{\circ} \mathrm{C}$ for $1 \mathrm{~h}$ (18). The signals were detected using an Odyssey infrared imaging system (Licor Biosciences). The specific protein expression levels were normalized against the expression of GAPDH.

Reverse transcription-quantitative polymerase chain reaction (RT-qPCR). Total RNA was extracted using a TRIzol kit (\#15596-026; Invitrogen Life Technologies), according to manufacturer's instructions. The RNA concentration was determined spectrophotometrically (Nanodrop 2000/2000C; Thermo Fisher Scientific, Inc.). The cDNA synthesis was performed using a cDNA Synthesis kit (\#04896866001; Roche) and $2 \mu \mathrm{g}$ total RNA. The PCR amplifications were quantified using a LightCycler 480 SYBR Green Master mix (\#04707516001; Roche) and the results were normalized against GAPDH. The relative mRNA expression levels of interleukin (IL)-1, IL-6 and tumor necrosis factor (TNF) $\alpha$ were assessed.

Statistical analysis. The data are expressed as the mean \pm standard error of the mean. The differences in the data between two groups was determined using Student's t-test. Comparisons between the groups were assessed by one-way analysis of variance followed by a Tukey's post-hoc test. $\mathrm{P}<0.05$ was considered to indicate a statistically significant difference.

\section{Results}

Effect of PA on the viability of LPS-treated H9c2 cells. The present study determined the effect of PA on $\mathrm{H} 9 \mathrm{c} 2$ cell viability using a CCK-8 assay. As shown in Fig. 1, following incubation with different concentrations of PA $(0.125,2.5,5$, 10 and $20 \mu \mathrm{M}$ ), with or without LPS for $12 \mathrm{~h}$, the $\mathrm{H} 9 \mathrm{c} 2$ cells in three concentrations of PA $(0.125,2.5$ and $5 \mu \mathrm{M})$ exhibited no differences in viability compared with the control cells. This indicated that neither $0.125,2.5$ or $5 \mu \mathrm{M}$ PA were not cytotoxic 
A

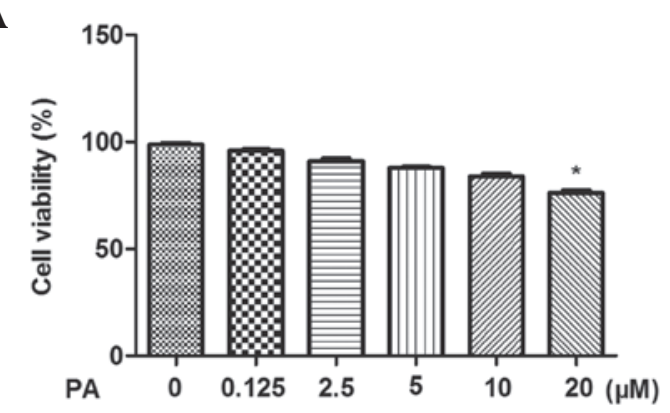

B

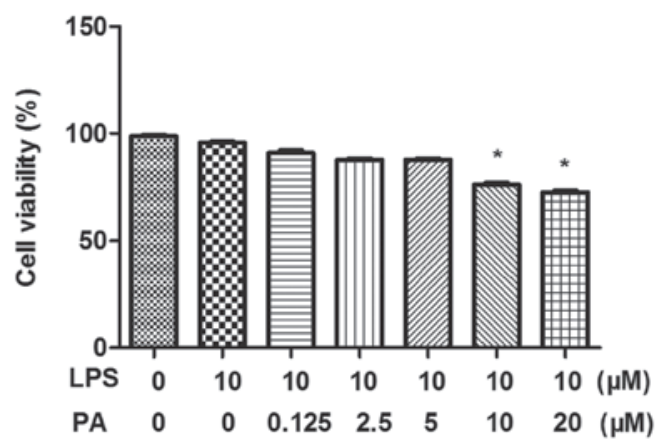

Figure 1. Effect of PA on cell viability using a Cell Counting kit-8 assay. Treatment with the indicated concentration of (A) PA alone and (B) PA with LPS $(10 \mu \mathrm{M})$ for $12 \mathrm{~h}$. PA treatment $0.125,2.5$ and $5 \mu \mathrm{M}$ exhibited no significant change in cell viability compared with the control group. Data are expressed as the mean \pm standard error of the mean. ${ }^{*} \mathrm{P}<0.05$ vs. control cells. LPS, lipopolysaccharide; PA, pachymic acid.
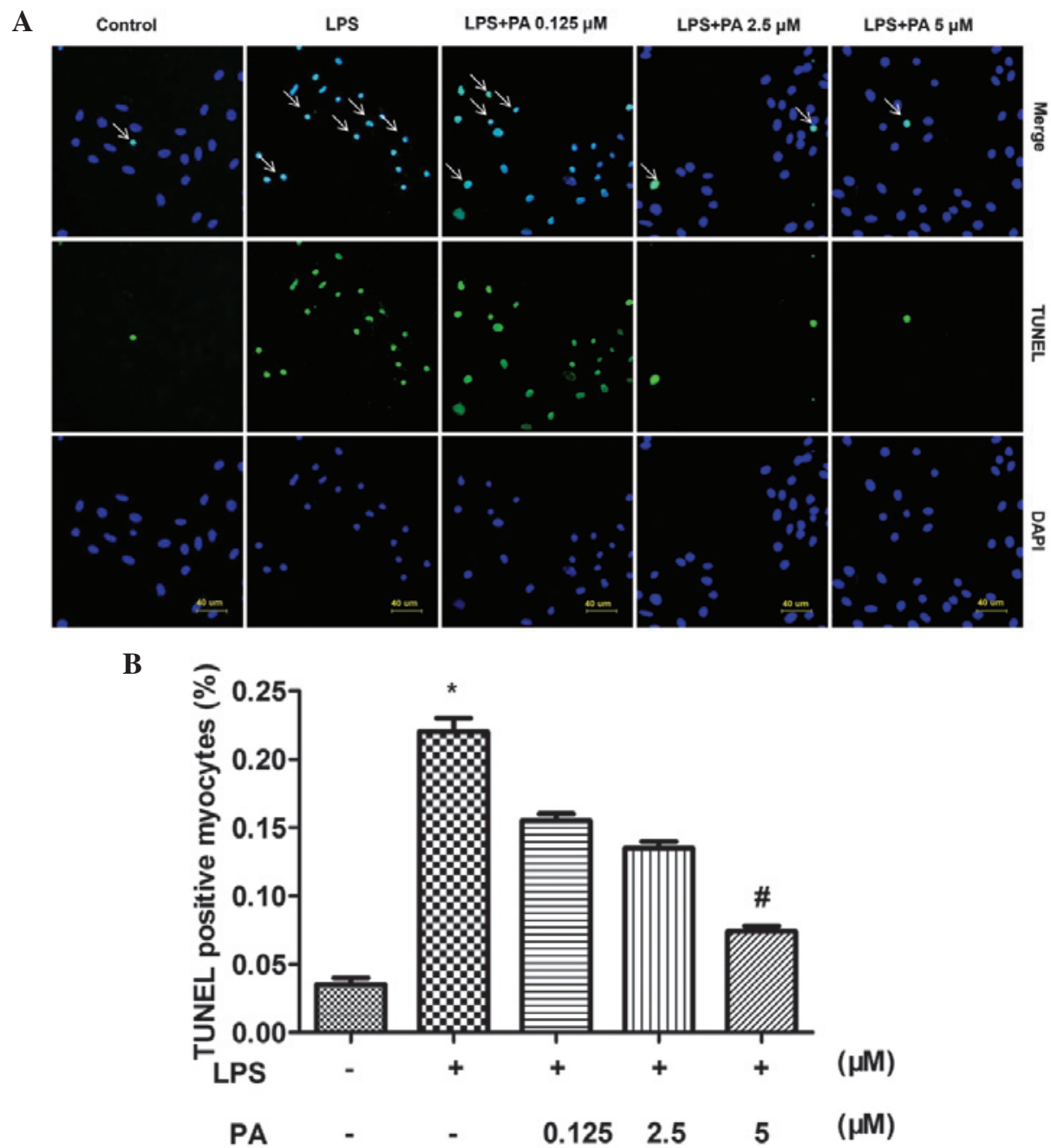

Figure 2. Effect of PA on apoptosis. PA $(0.125,2.5$ and $5 \mu \mathrm{M})$ attenuated cell apoptosis following stimulation with LPS (10 $\mu \mathrm{g} / \mathrm{ml})$ for $12 \mathrm{~h}$, demonstrated using ay TUNEL assay. (A) Representative images and (B) quantitative results in $\mathrm{H} 9 \mathrm{c} 2$ cardiomyocytes ( ${ }^{*} \mathrm{P}<0.05$, vs. control; ${ }^{*} \mathrm{P}<0.05$, vs. LPS). White arrows indicate TUNEL-positive cells. Data are expressed as the mean \pm standard error of the mean. PA, pachymic acid; LPS, lipopolysaccharide; TUNEL, terminal deoxynucleotidyl transferase dUTP nick end labeling.

to the H9c2 cells. Therefore, these three concentrations of PA were selected for use in the subsequent experiments.

Effect of PA on LPS-induced apoptosis in H9C2 cells. To investigate the protective role of PA in the LPS-induced apoptosis of H9c2 cells, TUNEL was used to stain the apoptotic nuclei. A noticeable increase in cardiomyocyte apoptosis was observed in the H9c2 cells induced by LPS, and treatment with PA reduced the LPS-induced apoptosis of cardiomyocytes (Fig. 2). 


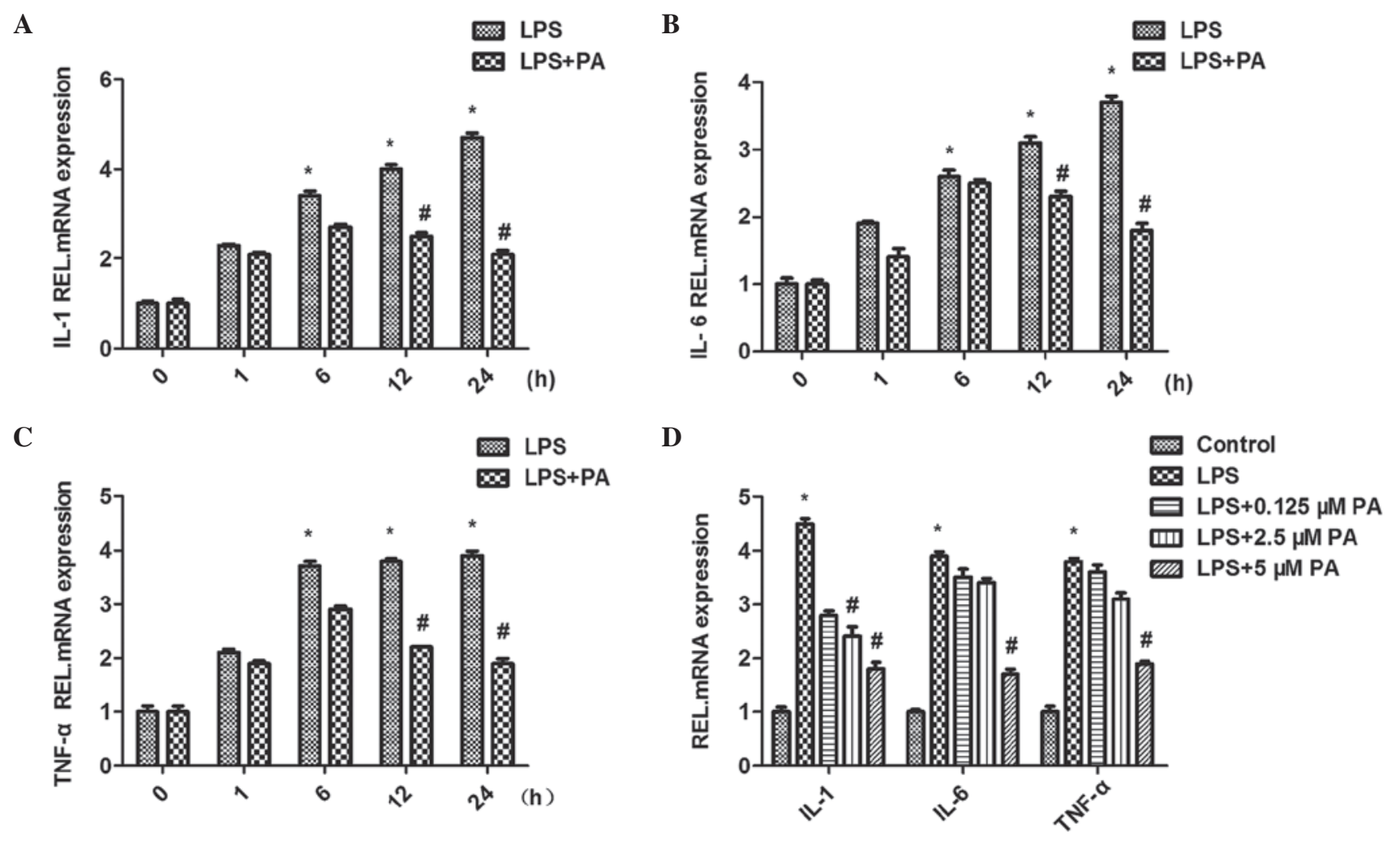

Figure 3. Effect of PA on the mRNA expression levels of inflammatory mediators, (A) IL-1, (B) IL-6 and (C) TNF- $\alpha$. (D) PA decreased LPS-induced expression levels of TNF- $\alpha$, IL-1 $\beta$ and IL- 6 in a concentration- and time-dependent manner $\left({ }^{*} \mathrm{P}<0.05\right.$, vs. control; ${ }^{\sharp} \mathrm{P}<0.05$, vs. LPS). Data are expressed as the mean \pm standard error of the mean. PA, pachymic acid; LPS, lipopolysaccharide; IL, interleukin; TNF, tumor necrosis factor; REL, relative.

A

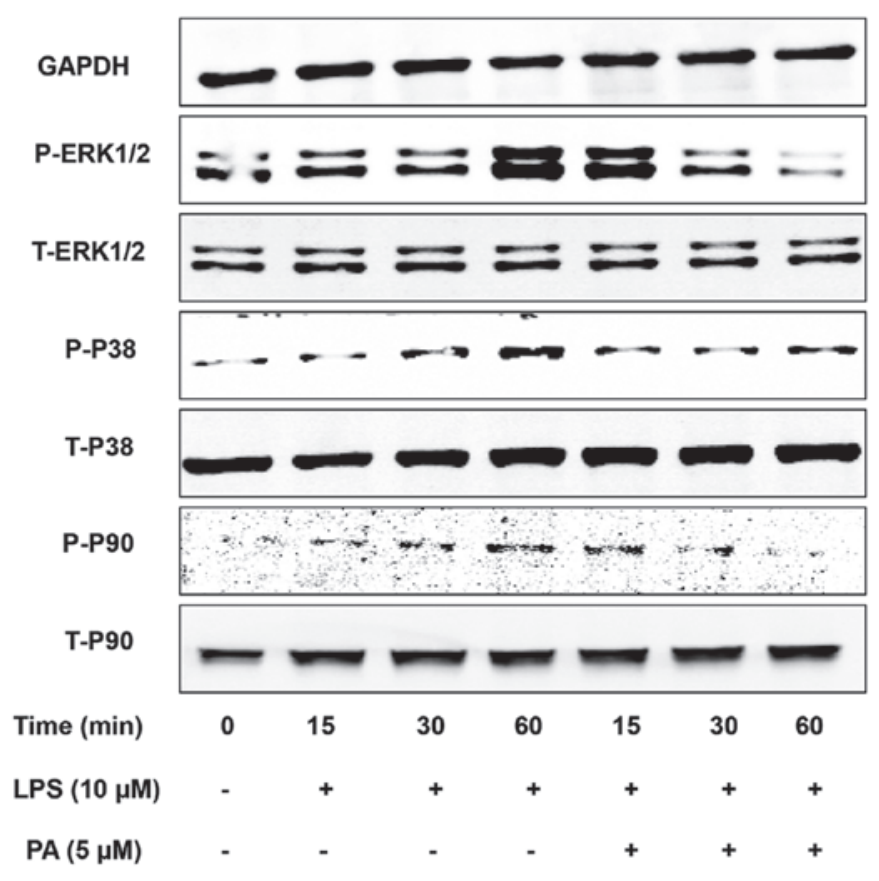

B

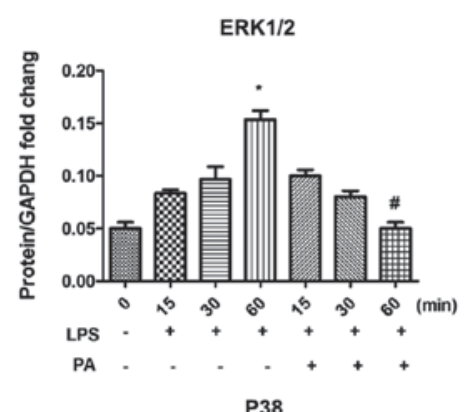

C

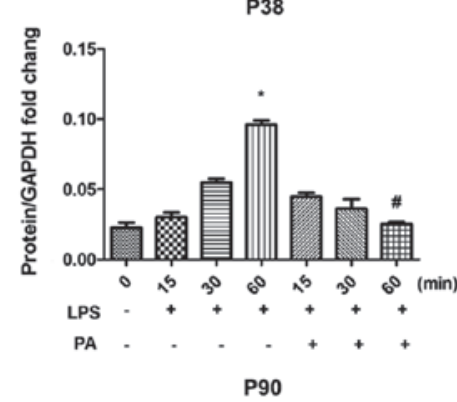

D

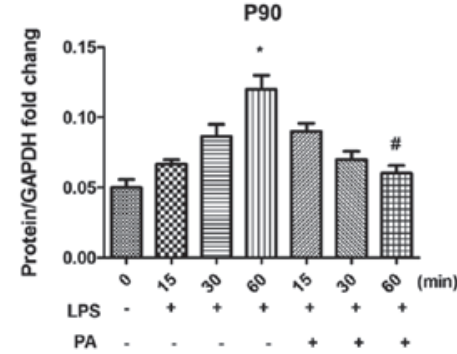

Figure 4.Effect of PA on the activation of signaling pathways. PA decreased the levels of p-Erk1/2,p38 and p90 in response to LPS (10 $\mu \mathrm{g} / \mathrm{ml})$. (A) Representative western blot analysis and (B-D) quantitative results ( $\mathrm{P}<0.05$ vs. control; ${ }^{*} \mathrm{P}<0.05$ vs. LPS). Data are expressed as the mean \pm standard error of the mean. p-, phosphorylated; PA, pachymic acid; LPS, lipopolysaccharide; Erk, extracellular-regulated kinase; GAPDH, glyceraldehyde-3-phosphate dehydrogenase. 
A

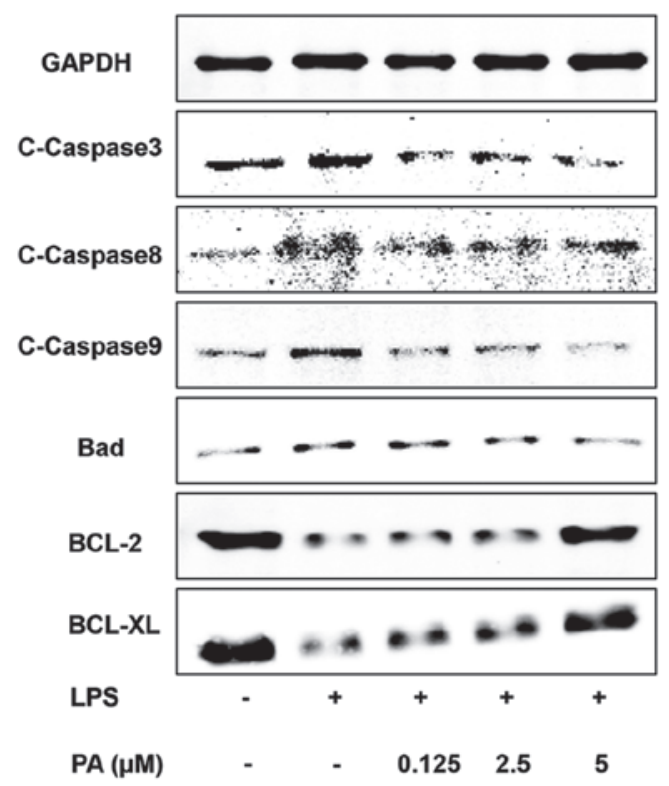

B

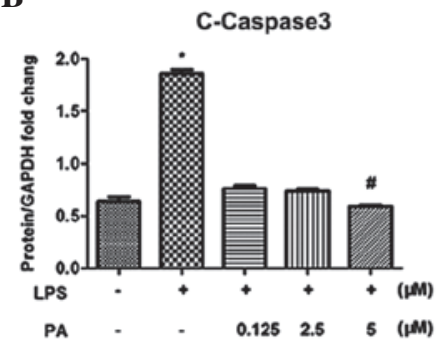

D

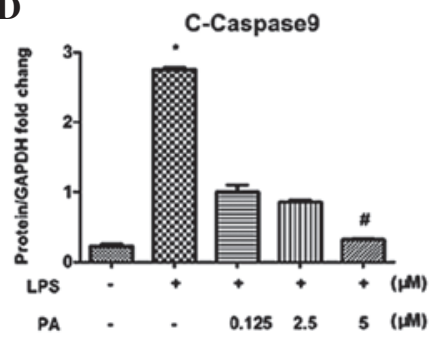

F

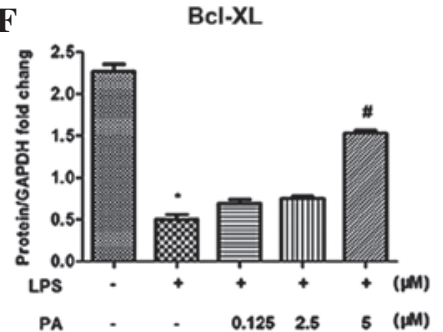

C

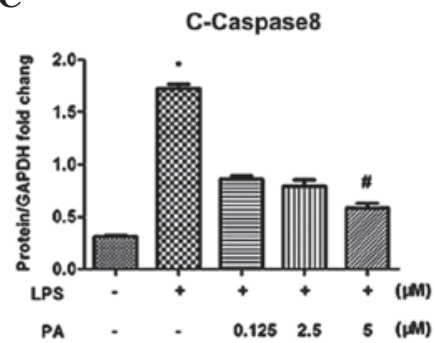

E
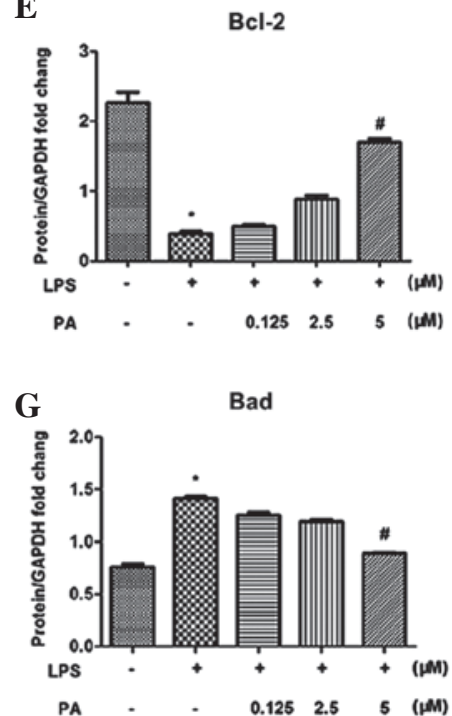

Figure 5. Effect of PA on the activation of apoptotic signaling pathways. PA decreased the levels of C-caspase-3, -8 and -9 . PA decreased the expression of Bad and increased the expression of of Bcl-2/Bcl-xL in response to LPS. (A) Representative western blotting and (B-G) quantitative results ('P<0.05, vs. control; ${ }^{\#} \mathrm{P}<0.05$, vs. LPS). Data are expressed as the mean \pm standard error of the mean. PA, pachymic acid; C-caspase, cleaved-caspase; LPS, lipopolysaccharide; Bcl, B-cell lymphoma; Bcl-X, Bcl-2 extra large; Bad, Bcl-2-associated death promoter; GAPDH, glyceraldehyde-3-phosphate dehydrogenase.

$P A$ attenuates the LPS-induced increased $M R N A$ expression levels of $I L-1, I L-6$ and TNF $\alpha$. Treatment of the H9c2 cardiomyocytes with LPS (10 $\mu \mathrm{M}$ for $12 \mathrm{~h})$ upregulated the mRNA expression levels of inflammatory markers, including IL-1, IL-6 and TNF $\alpha$. The induction of IL-1, IL-6 and TNF $\alpha$ in response to LPS $(10 \mu \mathrm{M})$ was restrained to different extents by different concentrations $(0.1,2.5$ and $5 \mu \mathrm{M})$ of PA. This indicated that treatment with PA led to a significant reduction in the mRNA expression levels of the IL-1, IL-6 and TNF $\alpha$ inflammatory markers in a concentration-dependent manner. The H9c2 cells were subsequently co-incubated with PA $(5 \mu \mathrm{M})$ and LPS $(10 \mu \mathrm{M})$ for different durations $(0,1,6,12$ and $24 \mathrm{~h})$. PA exhibited significant time-dependent inhibition of the LPS-induced activation of IL-1, IL-6 and TNFa (Fig. 3).

$P A$ regulates the apoptotic pathway and MAPK signaling. To investigate the molecular mechanisms underlying the anti-inflammatory and anti-apoptotic effects of PA, the apoptotic pathway and MAPK signaling pathways were examined. LPS increased the phosphorylation of extracellular-regulated kinase (Erk)1/2, p38 and p90, the downstream target of Erk1/2, and increased the activation of mediators of apoptosis, including caspase 3,8 and 9. Notably, PA significantly attenuated these effects, as shown in Figs. 4 and 5. In addition, the LPS-induced increased expression of pro-apoptotic B-cell lymphoma (Bcl)-2 family member, Bcl-2-associated agonist of cell death (Bad), was attenuated by PA, and the opposite result was observed for the anti-apoptotic Bcl-2 family members, Bcl-2 and Bcl-extra large (xL).

\section{Discussion}

The PA from $P$. cocos has been demonstrated in previous studies to possess anticancer and chemopreventive properties (12-14). The present study demonstrated that PA attenuated the LPS-induced apoptosis and inflammation in H9C2 cardiomyocytes. The data suggested that PA effectively attenuated LPS-stimulated apoptosis in the cardiomyocytes via suppression of the Erk and p38 signaling pathways. In addition, the present study demonstrated that the anti-inflammatory property of PA may be associated with the P38 signaling pathway.

Previous studies have suggested that the plasma concentrations of LPS are increased in patients with chronic heart failure, and controlling inflammation may assist in improving cardiac dysfunction and reducing sepsis-associated mortality (6). The inflammatory responses induced by LPS in cardiomyocytes lead to the activation of intracellular signaling pathways and transcription factors, and the induction of inflammatory mediators (19), including TNF- $\alpha$, IL-1 and IL-6. These mediators may be involved in the depression of cardiac function (20). PA, a lanostane-type triterpenoid and a major component of Poria cocos alcoholic extracts, has been reported to possess 
anti-inflammatory and anticancer properties in various models of cancer and inflammation (12,20-22). In the present study, PA exhibited anti-inflammatory ability. The expression levels of TNF- $\alpha$, IL-1 and IL- 6 in the H9c2 cells following LPS stimulation were examined, and the data revealed that the increased mRNA expression levels of these factors, induced by LPS, were attenuated following treatment with PA, indicating the anti-inflammatory property of PA in cardiomyocytes.

Loss of cardiomyocytes via apoptosis is considered a contributing factor in progressive deterioration of the hypertrophied left ventricle, ultimately leading to end-stage cardiomyopathy $(23,24)$. There has been increased interest in the effects of apoptosis on cardiomyocytes (25) following establishment that the cardioprotective effects of apoptosis are decreased by LPS. In the present study, PA downregulated the expression of Bad and upregulated the expression of Bcl-2 in the LPS-stimulated H9c2 cells, which may mediate the anti-apoptotic effect of PA. In addition, the increased expression levels of cleaved-caspase 3,8 and 9 were attenuated by treatment with PA, suggesting that PA attenuated the LPS-induced cardiomyocyte apoptosis, including endogenous and exogenous apoptosis. These findings indicated that, through its anti-apoptotic property, PA may be beneficial in certain heart diseases.

Based on the results of the present study that PA attenuated the LPS-induced inflammatory response and apoptosis in $\mathrm{H} 9 \mathrm{c} 2$ cells, the mechanisms underlying the beneficial effect of PA were further investigated. Kim et al demonstrated that PA inhibits oxidative stress induced inflammation in oral diseases treated with LPS by inhibiting the translocation of nuclear factor (NF)- $\kappa \mathrm{B}$ (12). Ling et al (13) revealed that PA suppresses the IL- $1 \beta$-induced activation of MAPKs and inhibits the IL-1-induced activation of MAPs and NF- $\kappa$ B signaling pathways in human A549 non-small cell lung cancer cells. It has also been observed that production of the IL-1, IL- 6 and TNF $\alpha$ inflammatory mediators, contribute to the LPS-induced activation of p38, which belongs to the MAPK family and is important in the inducting the inflammatory response and apoptosis (26). It is possible that the anti-inflammatory property of the PA may be closely associated with the p38 signaling pathway. It was demonstrated that LPS activated Erk1/2 and p38, which may lead to the phosphorylation and activation of p90. However, several previous studies have demonstrated that p90 activates Bad kinase, which is a proapoptotic Bcl-2 family protein involved in the mitochondrial pathway of apoptosis (27). Bad promotes apoptosis by binding to anti-apoptotic Bcl-2 proteins, including Bcl-xL and Bcl-2, in the outer mitochondrial membrane, which results in the release of cytochrome $c$ from the intermembrane space of the mitochondria into the cytoplasm This promotes the formation of an apoptosome complex, leading to the cleavage and activation of procaspase $9(28,29)$. Initiator caspases are closely coupled to pro-apoptotic signals, including Erk1/2 and p38. Once activated, these caspases cleave and activate downstream effector caspases, including caspase-3, which lead to apoptosis by cleaving cellular proteins. In the present study, PA treatment decreased the LPS-induced activation of Erk1/2 and p38, attenuated the activation of p90 and Bad, and decreased the expression levels of cleaved-caspase 3, 8 and 9, suggesting that PA attenuated LPS-induced apoptosis by inhibiting the Erk1/2 and p38 MAPK signaling pathways.

In conclusion, the present study demonstrated for the first time, to the best of our knowledge, the effect of PA on LPS-induced inflammation and apoptosis in H9c2 cardiomyocytes, which may negatively feedback to the Erk1/2 and p38 pathways. These results provided experimental evidence for the application of PA in the treatment of inflammatory injury of cardiovascular diseases.

\section{Acknowledgements}

This study was supported by the National Natural Science Foundation of China (no. 81270303).

\section{References}

1. Yang S, Li R, Qu X, Tang L, Ge G, Fang W, et al: Fosinoprilat alleviates lipopolysaccharide (LPS)-induced inflammation by inhibiting TLR4/NF- $\kappa \mathrm{B}$ signaling in monocytes. Cell Immunol 284: 182-186, 2013.

2. Cheng YC, Chen LM, Chang MH, Chen WK, Tsai FJ, Tsai $\mathrm{CH}$, et al: Lipopolysaccharide upregulates uPA, MMP-2 and MMP-9 via ERK1/2 signaling in H9c2 cardiomyoblast cells. Mol Cell Biochem 325: 15-23, 2009.

3. Yang Z, Liu Y, Deng W, Dai J, Li F, Yuan Y, et al: Hesperetin attenuates mitochondria-dependent apoptosis in lipopolysaccharide-induced H9C2 cardiomyocytes. Mol Med Rep 9: 1941-1946, 2014

4. Sammon JD, Klett DE, Sood A, et al: Sepsis after major cancer surgery. J Surg Res 193: 788-794, 2015.

5. Yang P, Han Y, Gui L, Sun J, Chen YL, Song R, et al: Gastrodin attenuation of the inflammatory response in $\mathrm{H} 9 \mathrm{c} 2$ cardiomyocytes involves inhibition of NF- $\mathrm{KB}$ and MAPKs activation via the phosphatidylinositol 3-kinase signaling. Biochem Pharmacol 85: 1124-1133, 2013.

6. Ronco C: Lipopolysaccharide (LPS) from the cellular wall of Gram-negative bacteria, also known as endotoxin, is a key molecule in the pathogenesis of sepsis and septic shock. Preface. Blood Purif 37 (Suppl 1): 1, 2014.

7. Song Y, Dou H, Gong W, Liu X, Yu Z, Li E, et al: Bis-N-norgliovictin, a small-molecule compound from marine fungus, inhibits LPS-induced inflammation in macrophages and improves survival in sepsis. Eur J Pharmacol 705: 49-60, 2013.

8. Alazawia W, Heath $\mathrm{H}$, et al: Stat2 loss leads to cytokine-independent, cell-mediated lethality in LPS-induced sepsis. Proc Natl Acad Sci USA 110, 8656-8661, 2013.

9. Hagiwara S, Iwasaka H, Matsumoto S and Noguchi T: Effect of enteral versus parenteral nutrition on LPS-induced sepsis in a rat model. J Surg Res 145: 251-256, 2008.

10. Hoesel LM, Niederbichler AD and Ward PA: Complement-related molecular events in sepsis leading to heart failure. Mol Immunol 44: 95-102, 2007.

11. Li YP, Huang J, Huang SG, et al: The compromised inflammatory response to bacterial components after pediatric cardiac surgery is associated with cardiopulmonary bypass-suppressed Toll-like receptor signal transduction pathways. J Crit Care 29: 312.e7-312.e13, 2014.

12. Kim TG, Lee YH, Lee NH, Bhattarai G, Lee IK, Yun BS, et al: The antioxidant property of pachymic acid improves bone disturbance against AH plus-induced inflammation in MC-3T3 E1 cells. J Endod 39: 461-466, 2013.

13. Ling H, Jia X, Zhang Y, Gapter LA, Lim YS, Agarwal R, et al: Pachymic acid inhibits cell growth and modulates arachidonic acid metabolism in nonsmall cell lung cancer A549 cells. Mol Carcinog 49: 271-282, 2010.

14. Gapter L, Wang Z, Glinski J and Ng KY: Induction of apoptosis in prostate cancer cells by pachymic acid from Poria cocos. Biochem Biophys Res Commun 332: 1153-1161, 2005.

15. Huang YC, Chang WL, Huang SF, Lin CY, Lin HC and Chang TC: Pachymic acid stimulates glucose uptake through enhanced GLUT4 expression and translocation. Eur J Pharmacol 648: 39-49, 2010. 
16. Du M, Huang K, Gao L, Yang L, Wang WS, Wang B, et al: Nardosinone protects $\mathrm{H} 9 \mathrm{c} 2$ cardiac cells from angiotensin II-induced hypertrophy. J Huazhong Univ Sci Technolog Med Sci 33: 822-826, 2013.

17. Guo R, Wu K, Chen J, Mo L, Hua X, Zheng D, et al: Exogenous hydrogen sulfide protects against doxorubicin-induced inflammation and cytotoxicity by inhibiting p38MAPK/NFKB pathway in $\mathrm{H} 9 \mathrm{c} 2$ cardiac cells. Cell Physiol Biochem 32: 1668-1680, 2013.

18. Chang YM, Tsai CT, Wang CC, Chen YS, Lin YM, Kuo CH, et al: Alpinate oxyphyllae fructus (Alpinia Oxyphylla Miq) extracts inhibit angiotensin-II induced cardiac apoptosis in $\mathrm{H} 9 \mathrm{c} 2$ cardiomyoblast cells. Biosci Biotechnol Biochem 77: 229-234, 2013.

19. Angeloni $\mathrm{C}$ and Hrelia S: Quercetin reduces inflammatory responses in LPS-stimulated cardiomyoblasts. Oxid Med Cell Longev 2012: 837104, 2012.

20. Fan MJ, Huang-Liu R, Shen CY, Ju DT, Lin YM, Pai P, et al: Reduction of TLR4 mRNA stability and protein expressions through inhibiting cytoplasmic translocation of huR transcription factor by E (2) and/or ER $\alpha$ in LPS-treated H9c2 cardiomyoblast cells. Chin J Physiol 57: 8-18, 2014.

21. Hong R, Shen MH, Xie XH and Ruan SM: Inhibition of breast cancer metastasis via PITPNM3 by pachymic acid. Asian Pac J Cancer Prev 13: 1877-1880, 2012.

22. Lee YH, Lee NH, Bhattarai G, Kim GE, Lee IK, Yun BS, et al: Anti-inflammatory effect of pachymic acid promotes odontoblastic differentiation via HO-1 in dental pulp cells. Oral Dis 19: 193-199, 2013.
23. Kazama K, Okada M, Yamawaki H, et al: Adipocytokine, omentin inhibits doxorubicin-induced $\mathrm{H} 9 \mathrm{c} 2$ cardiomyoblasts apoptosis through the inhibition of mitochondrial reactive oxygen species. Biochem Biophys Res Commun 457: 602-607, 2015.

24. Picatoste B, Ramirez E, Caro-Vadillo A, Iborra C, Ares-Carrasco S, Egido J, et al: Sitagliptin reduces cardiac apoptosis, hypertrophy and fibrosis primarily by insulin-dependent mechanisms in experimental type-II diabetes. Potential roles of GLP-1 isoforms. PLoS One 8: e78330, 2013.

25. Wang L, Huang H, Fan Y, Kong B, Hu H, Hu K, et al: Effects of downregulation of microRNA-181a on $\mathrm{H}_{2} \mathrm{O}_{2}$-induced $\mathrm{H} 9 \mathrm{c} 2$ cell apoptosis via the mitochondrial apoptotic pathway. Oxid Med Cell Longev: 1-16, 2014, 2014.

26. Wang W, Tang L, Li Y and Wang Y: Biochanin A protects against focal cerebral ischemia/reperfusion in rats via inhibition of p38-mediated inflammatory responses. J Neurol Sci 348: 121-125, 2015.

27. Lee KW, Kim SG, Kim HP, Kwon E, You J, Choi HJ, et al: Enzastaurin, a protein kinase $\mathrm{C}$ beta inhibitor, suppresses signaling through the ribosomal S6 kinase and bad pathways and induces apoptosis in human gastric cancer cells. Cancer Res 68: 1916-1926, 2008

28. Czabotar PE, Lessene G, Strasser A and Adams JM: Control of apoptosis by the BCL-2 protein family: implications for physiology and therapy. Nat Rev Mol Cell Biol 15: 49-63, 2014.

29. Laulier C and Lopez BS: The secret life of Bcl-2: Apoptosis-independent inhibition of DNA repair by $\mathrm{Bcl}-2$ family members. Mutat Res 751: 247-257, 2012. 